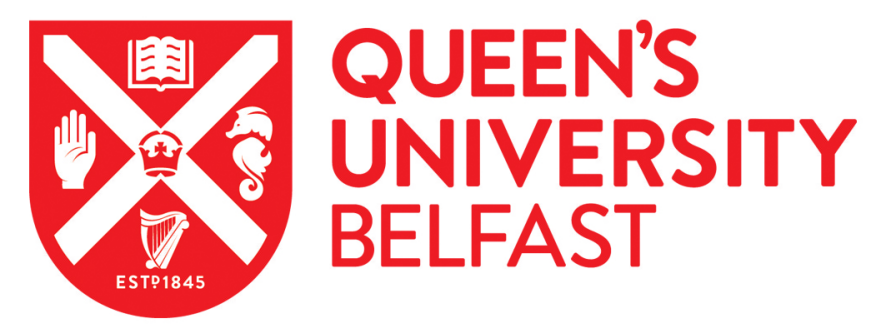

\title{
Effect of a complex intervention to improve post-vision screening referral compliance among pre-school children in China: A cluster randomized clinical trial
}

Zeng, Y., Han, X., Wang, D., Chen, S., Zheng, Y., Jiang, Y., Chen, X., Li, Y., Jin, L., Chen, Q., Liang, X., Zhang, X., Congdon, N., \& Liu, Y. (2020). Effect of a complex intervention to improve post-vision screening referral compliance among pre-school children in China: A cluster randomized clinical trial. EClinicalMedicine - published by THE LANCET, 19, [100258]. https://doi.org/10.1016/j.eclinm.2020.100258

Published in:

EClinicalMedicine - published by THE LANCET

Document Version:

Publisher's PDF, also known as Version of record

Queen's University Belfast - Research Portal:

Link to publication record in Queen's University Belfast Research Portal

\author{
Publisher rights \\ (c) 2020 The Authors. \\ This is an open access article published under a Creative Commons Attribution-NoDerivs License (https://creativecommons.org/licenses/by- \\ nd/4.0/), which permits reproduction and redistribute in any medium, provided the author and source are cited and any subsequent \\ modifications are not distributed.
}

\section{General rights}

Copyright for the publications made accessible via the Queen's University Belfast Research Portal is retained by the author(s) and / or other copyright owners and it is a condition of accessing these publications that users recognise and abide by the legal requirements associated with these rights.

\section{Take down policy}

The Research Portal is Queen's institutional repository that provides access to Queen's research output. Every effort has been made to ensure that content in the Research Portal does not infringe any person's rights, or applicable UK laws. If you discover content in the Research Portal that you believe breaches copyright or violates any law, please contact openaccess@qub.ac.uk. 
Research Paper

\title{
Effect of a complex intervention to improve post-vision screening referral compliance among pre-school children in China: A cluster randomized clinical trial
}

\author{
Yangfa Zeng ${ }^{\mathrm{a}, 1}$, Xiaotong Han ${ }^{\mathrm{a}, 1}$, Decai Wang ${ }^{\mathrm{a}, 1}$, Shida Chen ${ }^{\mathrm{a}}$, Yingfeng Zheng ${ }^{\mathrm{a}}$, Yuzhen Jiang ${ }^{\mathrm{b}}$, \\ Xiang Chen ${ }^{a}$, Yuting Li $^{a}$, Ling Jin ${ }^{a}$, Qianyun Chen ${ }^{a}$, Xiaoling Liang ${ }^{a}$, Xiulan Zhang ${ }^{a}$, \\ Nathan Congdon ${ }^{\mathrm{a}, \mathrm{c}}$, Yizhi Liu ${ }^{\mathrm{a}, *}$ \\ a State Key Laboratory of Ophthalmology, Zhongshan Ophthalmic Center, Sun Yat-sen University, Guangzhou, China \\ ${ }^{\mathrm{b}}$ UCL Institute of Ophthalmology, University College London and Moorfields Eye Hospital, London, UK \\ ' Centre for Public Health, Queen's University Belfast, Belfast, Northern Ireland, UK
}

\section{A R T I C L E I N F O}

\section{Article History:}

Received 8 October 2019

Revised 2 January 2020

Accepted 6 January 2020

Available online 4 February 2020

\section{Keywords:}

Eye disease screening

Vision screening

Pre-school students

Referral compliance

\begin{abstract}
A B S T R A C T
Background: We investigated whether specific appointments for quality-assured care could increase referral uptake, often low in China, in children's vision screening.

Methods: We randomized children aged 4-7 years in Yudu, Jiangxi, China, by school to Control (free schoolbased eye screening, parents of children failing screening recommended for further examination [usual practice]) or Intervention (identical examinations, with parents additionally provided with specific appointments for further examinations by quality-assured doctors at a designated local hospital). Both groups could select any hospital for referral exams, which were not free. Six months after screening, parents were interviewed on referral compliance at any hospital (primary outcome) and potential determinants. This trial is registered at the ClinicalTrials.gov, number NCT 03251456.

Findings: Among 9936 children at 63 schools randomized to Intervention (32 schools, 5053 [50.9\%] children) or Control (31 schools, 4883 [49.1\%] children), 1114 children (11.2\%) failed screening. Among 513 referred Intervention children (46.1\%, 32 schools, mean age 5.36 years, 53.0\% boys) and 601 referred Control children (53.9\%, 31 schools, mean age 5.30 years, 57.7\% boys), 104 (20.3\%) and 135 (22.5\%) were lost to follow-up respectively. Under Intention to Treat analysis, assuming children lost to follow-up were non-compliant, Intervention children had significantly higher compliance than Controls $(308 / 513=60.0 \%$ vs. 225/ $601=37.4 \%, P<0.001$ ). In regression models, Intervention group membership (Relative risk [RR] 1.53, 95\% confidence interval, 1.36-1.72), travel time to hospital (RR: 0.97, 0.95-0.999), baseline glasses wear (RR: 1.37, 1.17-1.60), strabismus (RR: 1.17, 1.01-1.36) and worse uncorrected vision (RR: 1.41, 1.03-1.92) were associated with compliance.

Interpretation: Providing specific appointments for quality-assured eye care improved referral compliance in this setting.
\end{abstract}

(C) 2020 Published by Elsevier Ltd. This is an open access article under the CC BY-NC-ND license. (http://creativecommons.org/licenses/by-nc-nd/4.0/)

\section{Introduction}

Screening is an essential public health service, aiming to identify high-risk populations and patients at early stages of disease. Timely and appropriate follow-up is imperative to achieve the goal of disease prevention and control. Children detected in vision screening program are often asymptomatic or at an early

\footnotetext{
* Corresponding author.

E-mail address: liuyizh@mail.sysu.edu.cn (Y. Liu).

1 These authors contributed equally.
}

clinical stage, thus post-screening clinical follow-up rates are often low $[1,2]$.

Good vision is important for children's health and well-being. The reported prevalence of amblyopia and ocular abnormalities among preschoolers were $1.6 \%$ and $2 \%$, respectively in recent large America studies [3,4]. High quality data are unavailable for China, but there is no reason to expect a lesser burden. Preschool vision screening has the potential to reduce the burden of visual impairment on society, and also to improve educational opportunities for children [5,6]. Many countries include preschool 


\section{Research in context}

\section{Evidence before this study}

We reviewed the interventions to improve referral compliance after eye screening among preschool children by searching PubMed and Google Web for original articles from July 4, 2019, to July 9, 2019, for articles published in any languages appearing after Jan 1, 1975 (See appendix for full search terms). Existing evidence suggests that interventions including education programs, follow-up phone calls, and including school nurses in the screening program may increase post-screening referral compliance among school children. Available publications generally suffered from small sample size, and no randomized controlled trials weres identified. Evidence is thus of weak-moderate quality.

\section{Added value of this study}

This large randomized controlled trial found that an inexpensive, complex intervention, including both specific appointments for follow-up examinations and quality-assured eye care, could significantly improve post-screening compliance $(308 / 513=60.0 \%$ vs. $225 / 601=37.4 \%, P<0.001)$ among preschool children in China. Our study found that doctors' perceived professional skills and level of the hospital were important considerations for parents in selection of facilities for follow-up. These findings highlight that both accessibility and perceived good quality of care are important in driving demand.

\section{Implications of all the available evidence}

Low-cost interventions focusing on improved access to care through scheduled appointments and enhanced perception of quality through quality-assured providers can significantly increase acceptance of eye examinations in low-resource settings. Further trials are needed elsewhere.

screening as part of regional or national government health care, but practices vary [7]. However in China, with 118.5 million preschool-aged children [8], there is no established national preschool screening or general practitioner-based referral system. Studies are needed to investigate appropriate vision screening and referral models for preschool children in China, and provide high-quality evidence to inform public policy.

A common short-coming of community screening programs is low referral compliance among individuals identified with disease [9]. Several reasons have been identified for lack of health-seeking behavior in Chinese population-based vision surveys, which include lack of knowledge about eye disease and its treatment, and concerns about the quality of locally-available care [10], with the latter proven particularly difficult to overcome [10,11]. A common feature in the Chinese health system, especially at rural, county-level facilities, is not to provide specific appointment dates, with patients choosing their own date to return and attempting to secure a place on the list of a potential examining physician.

In view of the lack of specific appointments and a documented concern over the quality of available care, the current study sought to assess the effect on post-screening referral compliance of a complex intervention, providing both a specific range of appointment dates and offering quality-assured eye care, in a vision screening program among preschool children in China.

\section{Methods}

\subsection{Study design}

The Yudu Preschool Study is a cluster-randomized, school-based trial conducted in Yudu county, Jiangxi province, China from August 2017 to June 2018. The study was approved by the Ethical Review Committee of the Zhongshan Ophthalmic Center (ZOC), Sun Yat-sen University, Guangzhou, China, and was conducted in accordance with the tenets of the Declaration of Helsinki. Before baseline data collection and screening, principals, and head teachers in participating kindergartens were fully informed of the study process and provided consent for participation. The parents or legal guardians of all participants provided written informed consent, and the trial was registered online (ClinicalTrials.gov ID: NCT 03251456) prior to participant enrollment. At the end of the screening, children and families received printed screening reports. All authors vouch for the accuracy and completeness of the data and for the fidelity to the trial protocol of this report. This study was reported according to the CONSORT extended guidelines.

\subsection{Participants, randomization and masking}

In 2017, there were 198 registered kindergartens in Yudu county with 31,812 children in attendance. After excluding kindergartens with fewer than 30 children, a total of 189 kindergartens (110 rural and 79 urban kindergartens) remained. A total of 63 kindergartens (32 rural and 31 urban) were selected randomly and assigned with a random number generating program (www.randomization.com) to the Intervention or Control group with a block size of four. Schoolbased cluster randomization was used in this study to prevent contamination, due to the enhanced feasibility of masking in this way, while the children's parents were aware whether they were part of the intervention or control arm and investigators, but not interviewers, were aware of cluster allocation. All children received vision screening per protocol, and those with abnormal screening results were provided with different referral suggestions in the Intervention and Control group (see below). After vision screening and before referral, the following children were further excluded from the study: (1) Those aged less than four or greater than seven years. (It is not uncommon for Chinese children aged seven years to still attend kindergarten, especially in rural areas). (2) Those whose parents failed to receive the screening report, based on a questionnaire interview.

\subsection{Procedures}

It is compulsory for preschool children in China to receive a VA screening and a general physical examination within the first semester of school as part of the national public health service program [12]. In addition to the routine screening performed by local health centers, we added free examinations as follows: refraction, examination of the red reflex in both eyes, cover-uncover test to detect strabismus and flashlight examination of the anterior segment, all of which were performed by local nurses, ocular technicians and ophthalmologists who had been trained by doctors from ZOC. General doctors and nurses from the local health center performed general physical examinations at the same time.

Uncorrected visual acuity (UCVA) was assessed by three trained ophthalmic nurses using standard logarithm of the minimum angle of resolution ( $\log$ MAR) VA charts with tumbling E optotypes at five meters per standardized protocol [13]. For children habitually wearing spectacles, presenting VA (wearing spectacles) was also measured. Refractive power in each eye without cycloplegia was measured using a handheld autorefractor (Mobile Vision Screener plusoptiX S12C, Nuremberg, Germany) by two trained technicians per standardized protocol. The red reflex was also examined using the handheld autorefractor. 
The cover-uncover test was performed by ophthalmologists to detect the presence of strabismus at both $40 \mathrm{~cm}$ and $5 \mathrm{~m}$. Tropias were categorized as esotropia, exotropia, or vertical tropia. The ophthalmologists also examined the appearance of both eyes, lids, corneas, and lenses with a handheld flashlight.

An abnormal screening result was defined as the presence of at least one of the following: (1) strabismus identified by the coveruncover test; (2) other ocular abnormality detected on flashlight examination of the anterior segment; or (3) finding of any of the below on handheld automated refraction, based on recommendations by the American Academy of Ophthalmology's PPP: [14]

1) Spherical power $\geq+1.75$ diopters (D) or $\leq-1.50 \mathrm{D}$ in either eye

2) Absolute inter-eye difference of spherical power $\geq 1.50 \mathrm{D}$

3) Cylindrical power $\geq 1.50 \mathrm{D}$ in either eye

4) Absolute inter-eye difference of cylindrical power $\geq 1.00 \mathrm{D}$

5) Inability to complete automated refraction in either eye

6) Abnormal red reflex during automated refraction.

In both study groups, we provided printed screening reports and informed parents of any abnormal results (see definition above) at the end of the screening. In the Control group, study personnel informed the parents of children with abnormal screening results that they should take their children to a hospital of their choice for further examination. In the Intervention group, parents were additionally provided with a referral card at the examination site with a medical appointment for further assessments within a designated time period at a referral center in a local secondary hospital (The People's Hospital of Yudu County), if they elected to go there. Parents in the Intervention group were also informed that doctors at the referral center had received professional training by doctors from ZOC, who would also be present at the referral center for management. Parents in both groups could select any hospital for referral exams, which were not free. Six months after the screening, all parents in both groups were interviewed for compliance assessment.

Two questionnaires were administered in this study. The vision history questionnaire was sent to each parent and collected by teachers on the next day when parents brought their children to school. Six months after the screening, parents were interviewed by phone with another questionnaire concerning factors potentially influencing compliance.

\subsection{Outcomes}

The primary outcome of the study was compliance with suggested referral eye examinations at any hospital within six months after receiving an abnormal screening report, in both the Intervention and Control groups. The numerator was number of participants who went for referral at any hospital within six months after receiving an abnormal screening report, and the denominator was the total number of participants who were recommend to receive referral eye examinations, whether they actually went for referral or not. Two independent interviewers from the local public health service who were familiar with the local language were trained before the study according to standardized questionnaire interview protocols. The interviewers were unaware of the study group assignment of participating children/families. Parents were asked whether they had taken their children for further examinations as suggested by telephone using the standard questionnaire above. If parents could not be contacted after three phone calls, teachers helped contact the parents and inform them of the phone interview. If parents still could not be contacted after a further phone call, home visits were conducted by two interviewers accompanied by local community health workers or government staff. Participants were deemed lost to follow-up if they could not be contacted using the above methods.

\subsection{Statistical analysis}

Based on the cluster-randomized design and an assumed compliance rate of $40 \%$ in the Control and $70 \%$ in the Intervention group [6], we determined that 30 schools ( 15 schools per group, with an average of 15 children expected to have abnormal vision screening test results at each), would provide $90 \%$ power at an alpha error of 0.05 , intra-class correlation (ICC) of 0.15 and variation in cluster sizes of 0.60 . Assumptions were based on previous screening programs in the area and our prior published trials [6]. Accounting for the stratified sampling by rural versus urban setting, a participation rate of $90 \%$ and loss to follow-up of $5 \%$, a total of 60 schools ( 30 per study group) was required.

Results were presented as median and inter quartile range (IQR) for continuous variables with non-normal distributions and number (proportions) for categorical variables. Spherical equivalent was defined as sphere plus half the negative cylinder. Statistical tests were performed for comparisons of baseline characteristics between the Intervention and Control groups: ordinal logistic regression for age ranging from 4 to7 years and Somers' $\mathrm{D}$ test for non-normally distributed continuous variables (travel time, uncorrected visual acuity and spherical equivalent refraction; logistic regression for binary variables: gender, urban dwelling, wearing glasses, ophthalmic history including head tilt present, squint or photophobia, ever had visual acuity testing, ever had ocular examination or refraction, ever diagnosed with eye disease, relative diagnosed with eye disease, and eye examination results: strabismus at $0.4 \mathrm{~m}$ or $5.0 \mathrm{~m}$ and any abnormality in routine examination.) All comparisons took cluster effects within kindergartens into account. The difference between groups in compliance rate was calculated using generalized linear models, with Poisson regression to estimate relative risk (RR) and 95\% confidence intervals $(\mathrm{CI})[15,16]$. All variables significant at the $P<0.10$ level in simple regression models were included in the multiple regression model. All statistical analyses were performed according to the trial protocol using a commercially available software package (Stata 13.1, StataCorp, College Station TX, USA).

For the intention to treat analysis, which required that all randomized participants to be included in analyses, we estimated the compliance rate in both groups in the most conservative fashion by assuming that all children lost to follow-up did not present for referral. For the risk factor analysis, we used multiple imputation in Stata to impute missing data, including for children lost to follow-up, using logistic regression models for binary variables and linear regression model for continuous variables, selecting the independent variables based on predictive value and availability of data. The multiple imputation approach created 20 copies of the data, in which missing values were imputed by chained equations. Final results were obtained by averaging these 20 datasets using Rubin's rules [17], which ensured that the standard errors for all regression coefficients took reflected uncertainty in the imputations as well as uncertainty in the estimation.

\subsection{Role of the funding source}

The funders of the study had no role in study design, data collection, data analysis, data interpretation, or writing of the report. The corresponding author had full access to all the data in the study and had final responsibility for the decision to submit for publication.

\section{Results}

A total of 63 schools with 9936 children underwent randomization; 32 kindergartens with 5053 children (50.9\%) were assigned to the Intervention and 31 kindergartens with 4883 children (49.1\%) to Control group. (Fig. 1) A total of 1114 children (11.2\%) had abnormal screening results, including 94 children (0.95\%) with astigmatism and $116(1.17 \%)$ with an ocular abnormality. Of the 540 children with abnormal vision screening results in the Intervention group, we 


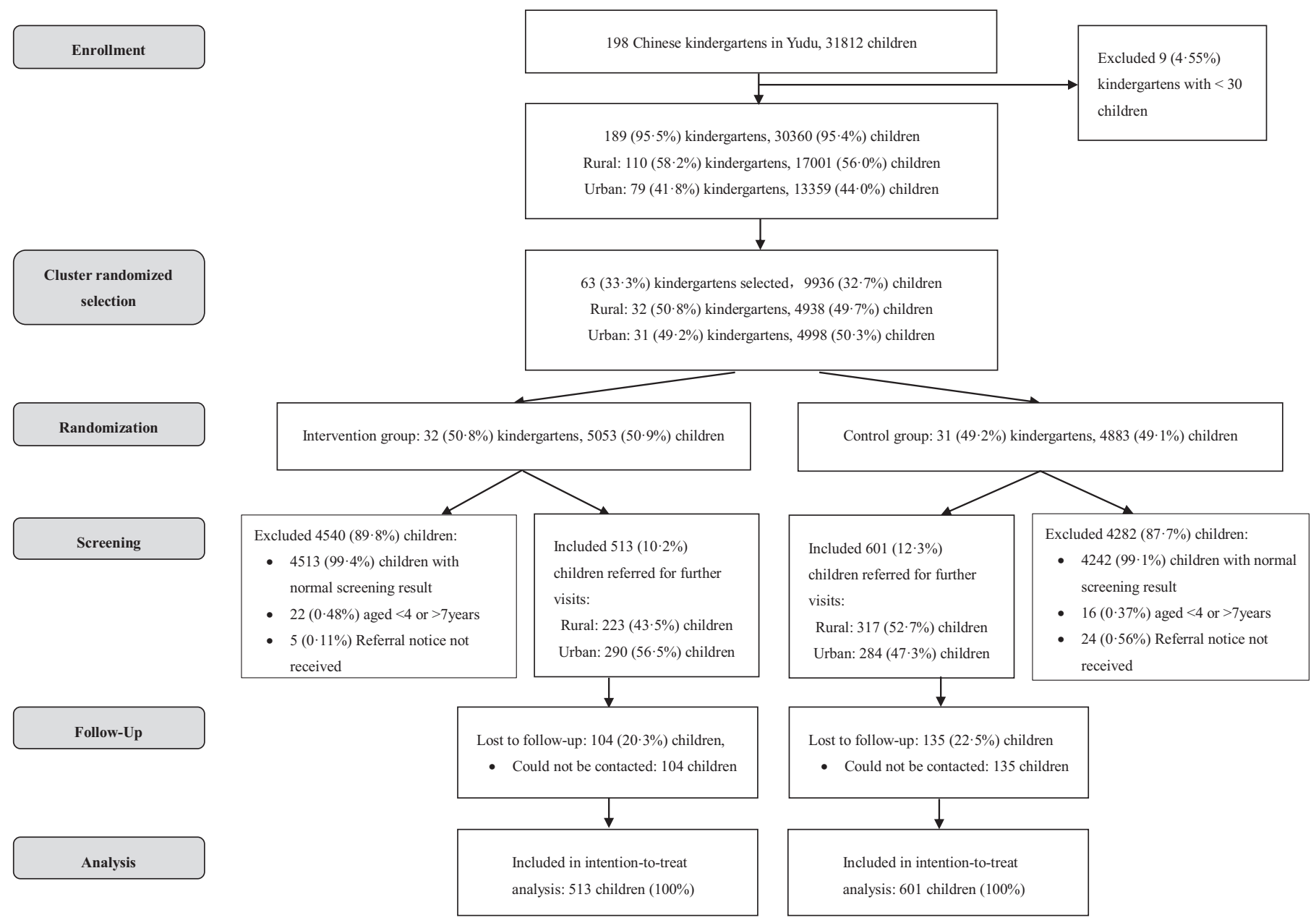

Fig. 1. Recruitment and flow of participants in the Yudu Preschool Study.

further excluded 22 children (4.07\%) aged less than four or greater than seven years and five children (0.93\%) whose parents failed to receive the screening report. Thus, a total of 513 Intervention children (95.0\%, 223 rural, and 290 urban) were eligible and included in the final analysis. Similarly, 601 (93.8\%, 317 rural and 284 urban) of the 641 children with abnormal screening results in the Control group were included in the final analysis. (Fig. 1)

Table 1 illustrates the baseline characteristics of children in the Intervention (mean [SD] age 5.36 [0.94] years) and Control group (5.30 [0.91] years). Children in the two study groups did not differ in age, gender, travel time to hospital, proportion of urban dwellers, or rate of baseline glasses wear. At the time of baseline screening, rates of detection of ocular abnormalities among children in the Intervention and Control groups were similar (Table 1), as were the mean logMAR UCVA (both 0.10 [6/7.5] [0-0.22], $P=0.288$ ). The median (inter-quartile range) spherical equivalent refraction in the betterseeing eye in the Control and Intervention groups were 0.50 $(0.00-1.13) \mathrm{D}$ and $0.38(0.00-1.00) \mathrm{D}$, respectively $(P=0.350)$. Six months after receiving an abnormal eye screening report and being informed of the need for further examination, 104 (20.3\%) and 135 (22.5\%) children were lost to follow-up in the Intervention and Control group, respectively. The baseline characteristics of children who completed and failed to complete follow-up did not differ significantly (Supplementary Table 1).

Under Intention to Treat analysis (assuming all children lost to follow-up were non-compliant), Intervention children had significantly higher compliance than Controls $(308 / 513=60.0 \%$ vs. $225 / 601=37.4 \%$, $P<0.001)$. Further analysis showed rural and urban-dwelling children had similar compliance rates in both the Control (35.3\% vs. $39.8 \%$, $\mathrm{RR}=1 \cdot 13,95 \% \mathrm{CI}: 0 \cdot 87-1 \cdot 45, P=0.361)$ and Intervention groups $(53.8 \%$ vs. $64.8 \%, \mathrm{RR}=1.20,95 \% \mathrm{CI}: 0.99-1.47, P=0.067)$. Membership in the Intervention group (RR: $1.53,1.36-1.72, P<0.001$ ), travel time from kindergarten to hospital (RR: 0.97, 0.95-0.999, $P=0.039$ ), wearing glasses at baseline (RR: $1.37,95 \% \mathrm{CI}: 1 \cdot 17-1.60, P<0.001$ ), signs of strabismus (RR: $1.17,95 \% \mathrm{CI}: 1.01-1.36, P=0.032$ ) and UCVA (RR: 1.41 , 95\%CI: $1.03-1.92, P=0.031)$ were significantly associated with successful referral compliance. (Table 2)

Based on the questionnaire interview at six months, among parents who took their children to hospital for further examination, the top two factors influencing choice of hospital were doctor's perceived professional skill $(61.6 \%$ and $68.6 \%$ in the Control and Intervention group, respectively), and level and size of the hospital (21.8\% and $15.4 \%$ in the Control and Intervention group, respectively). Among parents who did not take their children for further examination (342 children, 39\%), the top three barriers were the same in the Control and Intervention groups: lack of awareness, inconvenience and lack of access (39.1\%, $25.2 \%, 17.2 \%$ and $30.2 \%, 36.5 \%$ and $8.33 \%$ in the Control and Intervention group, respectively) (Supplementary Table 2).

\section{Discussion}

Disease screening is a basic public health service strategy worldwide, aimed at identifying individuals at increased risk of certain diseases in the general population for early intervention. Effective screening and early intervention for various ocular conditions have been proven to benefit patients' prognosis, reduce the cost of treatment, and improve the 
Table 1

Baseline characteristics of the study participants.

\begin{tabular}{|c|c|c|c|c|c|}
\hline Characteristics & $\begin{array}{l}\text { All } \\
(N=1114)\end{array}$ & $\begin{array}{l}\text { Control Group } \\
(N=601,53.9 \%)\end{array}$ & $\begin{array}{l}\text { Intervention Group } \\
(N=513,46.1 \%)\end{array}$ & $P$ value* & Missing data, $\mathrm{N}(\%)$ \\
\hline \multicolumn{6}{|l|}{ Demographics } \\
\hline Mean Age (SD), years & $5.32(0.93)$ & $5.30(0.91)$ & $5.36(0.94)$ & 0.529 & $0(0.00)$ \\
\hline Boys, No. (\%) & $619(55.6)$ & $347(57.7)$ & $272(53.0)$ & 0.090 & $0(0.00)$ \\
\hline Urban dwellers, No. (\%) & $574(51.5)$ & $284(47.3)$ & $290(56.5)$ & 0.529 & $0(0.00)$ \\
\hline Travel time from kindergarten to hospital, median (IQR), minutes & $20(10-55)$ & $30(10-55)$ & $20(10-45)$ & 0.638 & $0(0.00)$ \\
\hline Wearing glasses, No. (\%) & $44(3.98)$ & $22(3.69)$ & $22(4.32)$ & 0.710 & $8(0.72)$ \\
\hline \multicolumn{6}{|l|}{ Ophthalmic questionnaire, No. (\%) } \\
\hline Head tilt present & $84(8.27)$ & $46(8.20)$ & $38(8.35)$ & 0.929 & $98(8.80)$ \\
\hline Squint or photophobia & $79(7.78)$ & $40(7.13)$ & $39(8.57)$ & 0.512 & $98(8.80)$ \\
\hline Ever had visual acuity testing & $318(31.3)$ & $163(29.1)$ & $155(34.1)$ & 0.471 & $99(8.89)$ \\
\hline Ever had ocular examination or refraction & $188(18.6)$ & $92(16.4)$ & $96(21.2)$ & 0.386 & $102(9.16)$ \\
\hline Ever diagnosed with eye disease & $118(11.9)$ & $60(10.9)$ & $58(13.0)$ & 0.503 & $120(10.8)$ \\
\hline Relative diagnosed with eye disease & $54(5.47)$ & $27(4.95)$ & $27(6.11)$ & 0.572 & $127(11.4)$ \\
\hline \multicolumn{6}{|l|}{ Eye Examinations } \\
\hline \multicolumn{6}{|l|}{ Strabismus, No. (\%) } \\
\hline Present at $0.4 \mathrm{~m}$ & $77(6.98)$ & $45(7.56)$ & $32(6.30)$ & 0.413 & $11(0.99)$ \\
\hline Present at $5.0 \mathrm{~m}$ & $76(8.44)$ & $45(9.49)$ & $31(7.26)$ & 0.190 & $213(19.1)$ \\
\hline Uncorrected LogMAR visual acuity in the better-seeing eye, median (IQR) & $0.10(0-0.22)$ & $0.10(0-0.22)$ & $0.10(0-0.22)$ & 0.288 & $219(19.7)$ \\
\hline Spherical equivalent refraction in the better-seeing eye, median (IQR), diopters & $0.38(0.00-1.13)$ & $0.50(0.00-1.13)$ & $0.38(0.00-1.00)$ & 0.350 & $136(12.2)$ \\
\hline Any abnormality in routine examination, No. (\%) & $166(15.0)$ & $85(14.2)$ & $81(15.9)$ & 0.544 & $5(0.45)$ \\
\hline
\end{tabular}

IQR: Inter-quartile range.

* Ordinal logistic regression was used to compare age between the control and intervention groups; Somers' D test was used to compare travel time, uncorrected visual acuity and spherical equivalent refraction; Logistic regression was used for all binary variables. All comparisons took account of cluster effect within kindergartens.

Table 2

Intention to treat analysis of potential predictors of compliance with suggested eye examinations, adjusting for cluster effect within kindergartens.*

\begin{tabular}{|c|c|c|c|c|}
\hline & \multicolumn{2}{|c|}{ Simple regression } & \multicolumn{2}{|c|}{ Multiple regression $^{\dagger}$} \\
\hline & Relative Risk (95\% CI) & $P$-value & Relative risk $(95 \% \mathrm{CI})$ & $P$-value \\
\hline Intervention group (Control group as reference) & $1.56(1.37,1.78)$ & $<\mathbf{0 . 0 0 1}$ & $1.53(1.36,1.72)$ & $<0.001$ \\
\hline Age (Years) & $1.01(0.94,1.08)$ & 0.827 & & \\
\hline Male sex & $1.01(0.92,1.11)$ & 0.809 & & \\
\hline Urban dwelling & $1.18(1.01,1.39)$ & 0.040 & & \\
\hline Travel time to hospital, minutes & $0.96(0.93,0.99)$ & 0.011 & $0.97(0.95,0.999)$ & 0.039 \\
\hline Wearing glasses at baseline & $1.62(1.46,1.81)$ & $<0.001$ & $1.37(1.17,1.60)$ & $<0.001$ \\
\hline \multicolumn{5}{|l|}{ Results of ophthalmic examination/questionnaire } \\
\hline Head tilt & $1.33(1.15,1.53)$ & $<\mathbf{0 . 0 0 1}$ & $1.17(1.01,1.36)$ & $\mathbf{0 . 0 3 2}$ \\
\hline Squint or photophobia & $1.27(1.11,1.45)$ & $<\mathbf{0 . 0 0 1}$ & & \\
\hline Ever had visual acuity test & $1.16(1.04,1.29)$ & 0.009 & & \\
\hline Ever had ocular exam or refraction & $1.28(1.15,1.42)$ & $<\mathbf{0 . 0 0 1}$ & & \\
\hline Ever diagnosed with eye disease & $1.31(1.16,1.47)$ & $<\mathbf{0 . 0 0 1}$ & & \\
\hline Relative diagnosed with eye disease & $0.89(0.70,1.14)$ & 0.360 & & \\
\hline \multicolumn{5}{|l|}{ Strabismus present } \\
\hline At $0.4 \mathrm{~m}$ & $1.00(0.79,1.27)$ & 0.992 & & \\
\hline At $5.0 \mathrm{~m}$ & $0.82(0.63,1.06)$ & 0.122 & & \\
\hline Uncorrected visual acuity in the better-seeing eye & $1.88(1.38,2.57)$ & $<0.001$ & $1.41(1.03,1.92)$ & 0.031 \\
\hline Spherical equivalent refraction in the better-seeing eye, diopters & $0.99(0.93,1.05)$ & 0.651 & & \\
\hline Abnormality present on eye examination & $0.87(0.73,1.03)$ & 0.101 & & \\
\hline \multicolumn{5}{|c|}{ I: Confidence interval. The significance of the bold entries was shown by the p-values which were less than 0.05 . } \\
\hline \multirow{2}{*}{\multicolumn{5}{|c|}{$\begin{array}{l}\text { "Generalized linear models with Poisson regression was used to estimate the relative of follow-up compliance. } \\
\text { † Variables in the simple regression with } P \text { value }<0.05 \text { were included in the multiple regression. The variable "Urban residence" was not } \\
\text { included in the multiple regression analysis due to collinearity with "travel time from preschool to hospital". }\end{array}$}} \\
\hline & & & & \\
\hline \multicolumn{5}{|c|}{ The other variables on the ophthalmic questionnaire were not included in the multiple regression due to collinearity. } \\
\hline
\end{tabular}

cost-effectiveness of care. An impediment to optimizing the effectiveness and impact of eye disease screening remains the often-low rates of postscreening referral compliance [9,18]. Reported referral compliance after cancer screening ranged from $35 \%$ to $70 \%$, and compliance after the screening of cardiovascular diseases was also not ideal [19,20]. This problem also exists in children's eye screening, with reported follow-up rates ranging between $5 \%$ and $18 \%$ after a failed school vision test [2]. The importance of children's vision screening has been widely acknowledged: missing the opportunity for early diagnosis and intervention can lead to irreversible vision impairment or blindness in children, posing a significant burden to individuals, families, and society [14]. China has a large and increasing number of children with vision impairment [21], but lacks a practical and comprehensive screening and referral system. Proven models are needed.
We found that $11.2 \%$ of the children had abnormal screening results, which is higher than the reported prevalence of vision impairment or amblyopia in previous studies of preschool-aged children [3,22], suggesting a potentially increasing demand for eye health care in this age range. We found that providing specific appointments for quality-assured eye care at the conclusion of screening could significantly improve referral compliance in both urban and rural areas. Our finding that doctors' perceived professional skill and level of hospital were the most common reasons for choosing a follow-up facility is consistent with our main trial finding that availability of well-trained doctors can drive service uptake. The impact on the medical system of "celebrity doctors" and their particular appeal to Chinese patients has been well-described [23]. A low-cost intervention such as ours to certify the quality of local doctors is a far more sustainable and scalable alternative. 
The compliance rate for further eye examinations in our study was only $37.4 \%$ in the Control group, suggesting that merely informing parents of eye problems detected in their children leaves nearly two-thirds untreated. Very few previous trials have investigated compliance after vision screening in children, and studies other than trials report varying rates under different healthcare systems [24]. Alison et al. found that $34.4 \%$ and $48.1 \%$ of children failed to attend scheduled hospital eye services and local optometrist, respectively [25]. Tjiam et al. observed that $23 \%$ of children who had abnormal screening results did not present for further examinations as suggested [26]. Reported risk factors for non-adherence with post-eye screening referral include unawareness of the disease severity, poor access to care, financial burdens and inconvenient transport, similar to studies of screening for other diseases [27,28].

In our own study, longer travel time to the hospital decreased compliance, while the presence of symptomatic conditions and evidence of previous contact with the eye care system (e.g. astigmatism, poor vision or baseline glass wear) significantly increased it. Data from our questionnaire showed that lack of knowledge about eye disease was the most crucial barrier to referral compliance, indicating that education programs might be beneficial.

Previous studies of interventions to improve post-screening referral compliance have mostly focused on cancer screening [18,25], and few studies have reported on interventions to enhance compliance after eye screening in children $[19,28]$. One study in India reported a 23-step invention, which also included a specific appointment for children needing refraction, effective in increasing referral compliance [29] Another school-based study in the United States found that including school nurses in the screening pathway significantly improved referral compliance among children with abnormal screening results [2]. Education programs and follow-up phone calls have also been reported effective in enhancing post-screening referral compliance $[26,29]$. To the best of our knowledge, the current study is the only randomized clinical trial on referral compliance after eye screening in children.

Implications of our findings for program planners depend upon the practicality of this model for scale-up. Vision screening programs should not only inform patients of abnormal screening results, but also provide specific appointments, which can be easily accomplished, particularly when electronic medical record systems are available. Our simple intervention for quality certification is also scalable and sustainable, as it relied only on modest training and subsequent limited periods of training and oversight by junior doctors from tertiary facilities. Incentives to junior doctors to participate might include the opportunity for professional enhancement through training of trainers, and opportunities to participate in research, as was offered with the current paper.

Strengths of this study include the randomized, controlled design, a representative sample of kindergartens selected at random from a designated area, and a reasonable follow-rate in contacting parents. Limitations should also be acknowledged. It was not possible to mask the examiners, as patients in China expect feedback after examinations, including the follow-up plan which constituted our intervention, to be delivered directly by caregivers. A fifth of children was lost to follow-up in this study. To minimize the possible impact of this, we applied the most conservative possible assumption in our ITT analysis, namely that all such children defaulted on follow-up. We still observed significantly higher compliance rates in the Intervention compared to the Control group, which adds to the robustness of our conclusions. The sensitivity and specificity of vision screening for amblyopia and related risk factors were not assessed in the current study, as the primary outcome was post-screening compliance. A proper screening protocol is also of vital importance for vision screening among preschool children, and this warrants further investigation. Automated refraction without cycloplegia, as we performed here, is likely to result in some inaccuracies due to accommodation in young children. We felt this was acceptable for a screening examination, as cycloplegia can lead to high rates of parental refusal of examinations in China [30]. Further, these inaccuracies are unlikely to affect the reliability of our primary outcome of referral compliance. Lastly, this study only included Chinese children aged 4-7 years in a single county, and the application of our results to other populations can only be made with caution.

Despite these limitations, our study is one of the first designed to address a significant problem, that of poor follow-up referral after eye screening in children, particularly in a middle-income country. Our results suggest that providing specific appointments for qualityassured eye care in eye screening programs can significantly increase service uptake in this setting.

\section{Funding}

This study was funded by the National Natural Science Foundation of China (81530028; 81721003), Local Innovative and Research Teams Project of Guangdong Pearl River Talents Program; the State Key Laboratory of Ophthalmology, Zhongshan Ophthalmic Center, Sun Yat-sen University. Prof. Congdon is supported by the Ulverscroft Foundation (UK) and Director of Research with Orbis International, an organisation performing eye health work in low and middle income countries. The funding organizations had no role in the design or conduct of this research.

\section{Declaration of Competing Interest}

All authors declare that they have no competing interests.

\section{Supplementary materials}

Supplementary material associated with this article can be found in the online version at doi:10.1016/j.eclinm.2020.100258.

\section{References}

[1] Bruce A, Sanders T, Sheldon TA. Qualitative study investigating the perceptions of parents of children who failed vision screening at the age of 4-5 years. BMJ Paediatr Open 2018;2(1):e000307.

[2] Rodriguez E, Srivastava A, Landau M. Increasing screening follow-up for vulnerable children: a partnership with school nurses. Int J Environ Res Public Health 2018;15(8).

[3] Force USPST, Grossman DC, Curry SJ, et al. Vision screening in children aged 6 months to 5 years: US preventive services task force recommendation statement. JAMA 2017;318(9):836-44.

[4] Shakarchi AF, Collins ME. Referral to community care from school-based eye care programs in the United States. Surv Ophthalmol 2019.

[5] Jan C, Li SM, Kang MT, et al. Association of visual acuity with educational outcomes: a prospective cohort study. Br J Ophthalmol 2019.

[6] Ma X, Zhou Z, Yi H, et al. Effect of providing free glasses on children's educational outcomes in China: cluster randomized controlled trial. BMJ 2014;349:g5740.

[7] Abdolrahimzadeh S. Importance of vision screening in children regardless of socioeconomic status. Eye (Lond) 2012;26(3):478.

[8] Statistics NBo. China's sixth population census. 2010.

[9] Quigley HA, Park CK, Tracey PA, Pollack IP. Community screening for eye disease by laypersons: the Hoffberger program. Am J Ophthalmol 2002;133(3):386-92.

[10] Yin Q Hu A, Liang Y, et al. A two-site, population-based study of barriers to cataract surgery in rural china. Invest Ophthalmol Vis Sci 2009;50(3):1069-75.

[11] Liu T, Congdon N, Yan X, et al. A randomized, controlled trial of an intervention promoting cataract surgery acceptance in rural China: the Guangzhou Uptake of Surgery Trial (GUSTO). Invest Ophthalmol Vis Sci 2012;53(9):5271-8.

[12] The Basic Public Health Service Project Implementation Plan in Jiangxi Province China. 2016: http://www.jxwst.gov.cn/doc/2016/10/19/101883.shtml.

[13] Ferris 3rd FL, Kassoff A, Bresnick GH, Bailey I. New visual acuity charts for clinical research. Am J Ophthalmol 1982;94(1):91-6.

[14] Wallace DK, Morse CL, Melia M, et al. Pediatric eye evaluations preferred practice pattern(R): I. Vision screening in the primary care and community setting; II. Comprehensive ophthalmic examination. Ophthalmology 2018;125(1):P184-227.

[15] Zou GY, Donner A. Extension of the modified Poisson regression model to prospective studies with correlated binary data. Stat Methods Med Res 2013;22 (6):661-70.

[16] Zou G. A modified poisson regression approach to prospective studies with binary data. Am J Epidemiol 2004;159(7):702-6.

[17] P R. Multiple imputation of missing values. Stata J 2004;4(2):227-41. 
[18] Liang Y, Jiang J, Ou W, et al. Effect of community screening on the demographic makeup and clinical severity of glaucoma patients receiving care in Urban China. Am J Ophthalmol 2018;195:1-7.

[19] Selby K, Baumgartner C, Levin TR, et al. Interventions to improve follow-up of positive results on fecal blood tests: a systematic review. Ann Intern Med 2017; 167(8):565-75.

[20] Levitt NS, Puoane T, Denman CA, et al. Referral outcomes of individuals identified at high risk of cardiovascular disease by community health workers in Bangladesh, Guatemala, Mexico, and South Africa. Glob Health Action 2015;8:26318.

[21] Sun HP, Li A, Xu Y, Pan CW. Secular trends of reduced visual acuity from 1985 to 2010 and disease burden projection for 2020 and 2030 among primary and secondary school students in China. JAMA Ophthalmol 2015;133 (3):262-8.

[22] Xiao O, Morgan IG, Ellwein LB, He M. Refractive Error study in children study g. prevalence of amblyopia in school-aged children and variations by age, gender, and ethnicity in a multi-country refractive error study. Ophthalmology 2015;122(9):1924-31.

[23] Jiang Y, Luo L, Congdon N, Wang S, Liu Y. Who will be wielding the lancet for China's patients in the future? Lancet 2016;388(10054):1952-4.
[24] Messer DH, Mitchell GL, Twelker JD, Crescioni M, Group CS. Spectacle wear in children given spectacles through a school-based program. Optom Vis Sci 2012;89(1):19-26.

[25] Outhwaite ABL. Uptake, referral and attendance: results from an inner city school based vision screening programme. Br Ir Orthopt J 2013;10:41-5.

[26] Tjiam AM, Groenewoud JH, Passchier J, et al. Determinants and outcome of unsuccessful referral after positive screening in a large birth-cohort study of population-based vision screening. J AAPOS 2011;15(3):256-62.

[27] Keenum Z, McGwin Jr. G, Witherspoon CD, Haller JA, Clark ME, Owsley C. Patients' adherence to recommended follow-up eye care after diabetic retinopathy screening in a publicly funded county clinic and factors associated with follow-up eye care use. JAMA Ophthalmol 2016;134(11):1221-8.

[28] Bastani R, Yabroff KR, Myers RE, Glenn B. Interventions to improve follow-up of abnormal findings in cancer screening. Cancer 2004;101(5 Suppl):1188-200.

[29] Narayanan A, Ramani KK. Effectiveness of interventions in improving compliance to spectacle wear and referral in school vision screening. Clin Exp Optom 2018;101(6):752-7.

[30] Yi H, Zhang H, Ma X, et al. Impact of free glasses and a teacher incentive on children's use of eyeglasses: a cluster-randomized controlled trial. Am J Ophthalmol 2015;160(5) 889-96 e1. 